ISSN 1855-3966 (printed edn.), ISSN 1855-3974 (electronic edn.)

ARS MATHEMATICA CONTEMPORANEA 21 (2021) \#P1.01

https://doi.org/10.26493/1855-3974.1840.6e0

(Also available at http://amc-journal.eu)

\title{
Realisation of groups as automorphism groups in permutational categories
}

\author{
Gareth A. Jones * \\ School of Mathematical Sciences, University of Southampton, \\ Southampton SO17 1BJ, UK
}

Received 31 October 2018, accepted 18 July 2020, published online 10 August 2021

\begin{abstract}
It is shown that in various categories, including many consisting of maps or hypermaps, oriented or unoriented, of a given hyperbolic type, or of coverings of a suitable topological space, every countable group $A$ is isomorphic to the automorphism group of uncountably many non-isomorphic objects, infinitely many of them finite if $A$ is finite. In particular, the latter applies to dessins d'enfants, regarded as finite oriented hypermaps.
\end{abstract}

Keywords: Permutation group, centraliser, automorphism group, map, hypermap, dessin d'enfant.

Math. Subj. Class. (2020): 05C10, 14H57, 20B25, 20B27, 52B15, 57M10

\section{Introduction}

In 1939 Frucht published his celebrated theorem [15] that every finite group is isomorphic to the automorphism group of a finite graph; in 1960, by allowing infinite graphs, Sabidussi [46] extended this result to all groups. Similar results have been obtained, realising all finite groups (or in some cases all groups, or all countable groups) as automorphism groups of various other mathematical structures. Examples include the following, in chronological order: distributive lattices, by Birkhoff [4] in 1946; regular graphs of a given degree, by Sabidussi [45] in 1957; Riemann surfaces, by Greenberg [18, 19] in 1960 and 1973; projective planes, by Mendelsohn [37] in 1972; Steiner triple and quadruple systems, by Mendelsohn [38] in 1978; fields, by Fried and Kollár [14] in 1978; matroids of rank 3, by Babai [1] in 1981; oriented maps and hypermaps, by Cori and Machì [10] in 1982; finite volume hyperbolic manifolds of a given dimension, by Belolipetsky and Lubotzky [3] in

\footnotetext{
*The author is grateful to Ernesto Girondo, Gabino González-Diez and Rubén Hidalgo for discussions about dessins d'enfants which motivated this work, and also to Ashot Minasyan, Egon Schulte, David Singerman and Alexander Zvonkin for some very helpful comments.

E-mail address: g.a.jones@maths.soton.ac.uk (Gareth A. Jones)
} 
2005; abstract polytopes, by Schulte and Williams [47] in 2015 and by Doignon [12] in 2016. Babai has given comprehensive surveys of this topic in $[1,2]$.

In many of these cases, each group is represented as the automorphism group of not just one object, but infinitely many non-isomorphic objects. The aim of this paper is to obtain results of this nature for certain 'permutational categories', introduced and discussed in [24]. These are categories $\mathfrak{C}$ which are equivalent to the category of permutation representation of some 'parent group' $\Gamma=\Gamma_{\mathfrak{C}}$ : thus each object $\mathcal{O}$ in $\mathfrak{C}$ can be identified with a permutation representation $\theta: \Gamma \rightarrow S:=\operatorname{Sym}(\Omega)$ of $\Gamma$ on some set $\Omega$, and the morphisms $\mathcal{O}_{1} \rightarrow \mathcal{O}_{2}$ can be identified with the functions $\Omega_{1} \rightarrow \Omega_{2}$ which commute with the actions of $\Gamma$ on the corresponding sets $\Omega_{i}$. They include the categories of maps or hypermaps on surfaces, oriented or unoriented, and possibly of a given type. Other examples include the category of coverings of a 'suitably nice' topological space; this includes the category of dessins d'enfants, regarded as finite coverings of the thrice-punctured sphere, or equivalently as finite oriented hypermaps.

The automorphism group $\operatorname{Aut}_{\mathfrak{C}}(\mathcal{O})$ of an object $\mathcal{O}$ in a permutational category $\mathfrak{C}$ is identified with the centraliser $C:=C_{S}(G)$ in $S$ of the monodromy group $G:=\theta(\Gamma)$ of $\mathcal{O}$. Now $\mathcal{O}$ is connected if and only if $G$ is transitive on $\Omega$, as we will assume throughout this paper. Such objects correspond to conjugacy classes of subgroups of $\Gamma$, the pointstabilisers. An important result is the following:

Theorem 1.1. $\operatorname{Aut}_{\mathfrak{C}}(\mathcal{O}) \cong N_{G}(H) / H \cong N_{\Gamma}(M) / M$, where $H$ and $M$ are the stabilisers in $G$ and $\Gamma$ of some $\alpha \in \Omega$, and $N_{G}(H)$ and $N_{\Gamma}(M)$ are their normalisers.

There are analogous results in various contexts, ranging from abstract polytopes to covering spaces, which can be regarded as special cases of Theorem 1.1. Proofs of this result for particular categories can be found in the literature: for instance, in [28] it is deduced for oriented maps from a more general result about morphisms in that category; in [29, Theorem 2.2 and Corollary 2.1] a proof for dessins is briefly outlined; similar results for covering spaces are proved in [35, Appendix] and [39, Theorem 81.2], and for abstract polytopes in [36, Propositions 2D8 and 2E23(a)]. Theorem 1.1 follows immediately from the following 'folklore' result, proved in [26, Theorem 2(1)] (see also [44, Theorem 3.2]):

Theorem 1.2. Let $G$ be a transitive permutation group on a set $\Omega$, with $H$ the stabiliser of some $\alpha \in \Omega$, and let $C:=C_{S}(G)$ be the centraliser of $G$ in the symmetric group $S:=\operatorname{Sym}(\Omega)$. Then $C \cong N_{G}(H) / H$.

Of course, finite objects in any category have finite automorphism groups. In most of the permutational categories we will consider, the parent groups are finitely generated, so by Theorem 1.1 the automorphism groups of connected objects are all countable. Let us define a category $\mathfrak{C}$ to be countably (resp. finitely) abundant if every countable (resp. finite) group $A$ is isomorphic to $\operatorname{Aut}_{\mathfrak{C}}(\mathcal{O})$ for some connected object (resp. finite connected object) $\mathcal{O}$ in $\mathfrak{C}$. Let us define $\mathfrak{C}$ to be countably (resp. finitely) superabundant if there are $2^{\aleph_{0}}$ (resp. $\aleph_{0}$ ) isomorphism classes of such objects $\mathcal{O}$ realising each $A$.

In the case of a permutational category $\mathfrak{C}$, these properties follow immediately from Theorem 1.1 if the associated parent group $\Gamma$ has the corresponding abundance properties, namely that every countable group $A$ is isomorphic to $N_{\Gamma}(M) / M$ for the required number of conjugacy classes of subgroups $M$ of $\Gamma$, and these can be chosen to have finite index in $\Gamma$ if $A$ is finite. If $\Gamma$ is finitely generated, then the cardinalities $2^{\aleph_{0}}$ (resp. $\aleph_{0}$ ) are the best that can be achieved, since they are upper bounds on the number of conjugacy classes of 
subgroups (resp. subgroups of finite index) in $\Gamma$, and hence on the number of isomorphism classes of objects (resp. finite objects) available.

We will be mainly concerned with permutational categories consisting of maps and hypermaps of various types $(p, q, r)$, where $p, q, r \in \mathbb{N} \cup\{\infty\}$. For these, the parent groups are either extended triangle groups $\Delta[p, q, r]$, generated by reflections in the sides of a triangle with internal angles $\pi / p, \pi / q$ and $\pi / r$, or (for subcategories of oriented objects) their orientation-preserving subgroups, the triangle groups $\Delta(p, q, r)$. We say that a triple $(p, q, r)$ is spherical, euclidean or hyperbolic as $p^{-1}+q^{-1}+r^{-1}>1,=1$ or $<1$ respectively (where by convention we take $\infty^{-1}=0$ ), so that these groups act on the sphere, euclidean plane, or hyperbolic plane. We will call the triple cocompact if these groups act cocompactly, or equivalently $p, q, r \in \mathbb{N}$. We will use Theorem 1.1 to prove:

\section{Theorem 1.3.}

(a) If $(p, q, r)$ is a hyperbolic triple, where $p, q, r \in \mathbb{N} \cup\{\infty\}$, then the groups $\Delta(p, q, r)$ and $\Delta[p, q, r]$, together with their associated categories of oriented hypermaps and of all hypermaps of type $(p, q, r)$, are finitely superabundant.

(b) If, in addition, $(p, q, r)$ is not cocompact then these groups and categories are all countably superabundant.

By contrast, if we take $\Gamma$ to be a Tarski monster [42], an infinite group in which every subgroup $M \neq \Gamma, 1$ has order $p$ for some (very large) prime $p$, then $N_{\Gamma}(M)=M$ for each $M \neq 1$, and hence the only groups realised as automorphism groups in the corresponding category are 1 and $\Gamma$.

The spherical and euclidean triples must be excluded from Theorem 1.3 since the corresponding triangle groups are either finite or solvable, so the same restriction applies to the automorphism groups of connected objects in the associated categories. By taking $p=r=\infty$ and $q=2$ or $\infty$ we see that the categories $\mathfrak{M}$ and $\mathfrak{H}$ of all maps and hypermaps, together with their subcategories $\mathfrak{M}^{+}$and $\mathfrak{H}^{+}$of oriented maps and hypermaps, satisfy Theorem 1.3. In the case of $\mathfrak{M}^{+}$and $\mathfrak{H}^{+}$, Cori and Machì [9] showed in 1982 that every finite group arises as an automorphism group; they considered only finite groups, but their proof extends to countable groups. In fact, by Theorem 1.3(a) the category of Grothendieck's dessins d'enfants [20] of any given hyperbolic type is finitely superabundant. Of course these categories are not countably abundant. Nevertheless, in $\S 8$ we will prove a result, based on work of Conder [7, 8] on alternating and symmetric quotients of triangle groups, to support the following conjecture:

Conjecture 1.4. The non-cocompactness condition can be omitted from Theorem 1.3(b), so that the triangle groups $\Delta(p, q, r)$ and $\Delta[p, q, r]$ of any hyperbolic type, and their associated categories, are countably superabundant.

The proof of Theorem 1.3 is divided into several cases, depending on the particular group $\Gamma=\Gamma_{\mathfrak{C}}$ involved and whether we wish to realise countable or finite groups. In each case we construct a primitive permutation representation of $\Gamma$, of infinite or unbounded finite degree, such that a point stabiliser $N$ has an epimorphism onto a free group of countably infinite or unbounded finite rank, and hence onto an arbitrary countable or finite group $A$. By arranging that the kernel $M$ is not normal in $\Gamma$ we see from the maximality of $N$ in $\Gamma$ that $N_{\Gamma}(M) / M=N / M \cong A$, so Theorem 1.1 gives $\operatorname{Aut}_{\mathfrak{C}}(\mathcal{O}) \cong A$ for the object $\mathcal{O} \in \mathfrak{C}$ corresponding to $M$. Variations in the constructions yield $2^{\aleph_{0}}$ or $\aleph_{0}$ conjugacy 
classes of such subgroups $M \leq \Gamma$, and hence that number of objects $\mathcal{O}$ realising $A$. These objects are regular coverings, with covering group $A$, of the object $\mathcal{N} \cong \mathcal{O} / \operatorname{Aut}_{\mathfrak{C}}(\mathcal{O})$ in $\mathfrak{C}$ corresponding to $N$ and its conjugates.

In fact a deep result of Belolipetsky and Lubotzky [3, Theorem 2.1] implies finite superabundance for every finitely generated group which is large, that is, has a subgroup of finite index with an epimorphism onto a non-abelian free group. This applies to every nonelementary finitely generated Fuchsian group, and in particular to every hyperbolic triangle group, as in Theorem 1.3(a). However, the proof of [3, Theorem 2.1] is long, delicate and non-constructive, so here we offer a shorter, more direct argument, specific to the context of this paper in using maps and hypermaps.

One should not confuse countable abundance with the SQ-universality of a group $\Gamma$, a concept introduced by P. M. Neumann in [41], and proved there for (among others) all hyperbolic triangle groups and extended triangle groups: this requires that every countable group is isomorphic to a subgroup of a quotient of $\Gamma$, that is, to $N / M$ where $M \leq N \leq \Gamma$ and $M$ is normal in $\Gamma$, so that $N_{\Gamma}(M)=\Gamma$, while countable abundance requires that $N_{\Gamma}(M)=N$. In terms of permutational categories, SQ-universality of the parent group $\Gamma$ means that every countable group $A$ is embedded in the automorphism group of some regular object $\mathcal{O}$ (one with a transitive monodromy group $G$, so that $M$ is normal in $\Gamma$ and $\left.\operatorname{Aut}_{\mathfrak{C}}(\mathcal{O}) \cong \Gamma / M \cong G\right)$, whereas countable abundance means that $A$ is isomorphic to the automorphism group of some object, not necessarily regular. Both properties mean that any phenomenon exhibited by some countable group, no matter how exotic or pathological, can be realised within $\Gamma$, and hence within $\mathfrak{C}$ : see [23] for some examples where $\mathfrak{C}=\mathfrak{H}^{+}$.

Soon after this paper was submitted, a very interesting paper [5] by Bottinelli, Grave de Peralta and Kolpakov appeared on the arXiv. It independently introduces some of the concepts and proves some of the results presented here: for instance their concept of a 'telescopic group' coincides with our notion of finite abundance, and they prove this for all free products of cyclic groups (except $C_{2} * C_{2}$ ). However, their methods of construction differ substantially from ours, and they obtain asymptotic estimates for the number of finite objects realising a given finite group as their automorphism group, a topic not considered here.

\section{Permutational categories}

Following [24], let us define a permutational category $\mathfrak{C}$ to be a category which is equivalent to the category of permutation representations $\theta: \Gamma \rightarrow S:=\operatorname{Sym}(\Omega)$ of a parent group $\Gamma=\Gamma_{\mathfrak{C}}$. We then define the automorphism group $\operatorname{Aut}(\mathcal{O})=\operatorname{Aut}_{\mathfrak{C}}(\mathcal{O})$ of an object $\mathcal{O}$ in $\mathfrak{C}$ to be the group of all permutations of $\Omega$ commuting with the action of $\Gamma$ on $\Omega$; thus it is the centraliser $C_{S}(G)$ of the monodromy group $G=\theta(\Gamma)$ of $\mathcal{O}$ in the symmetric group $S$. In this paper we will restrict our attention to the connected objects $\mathcal{O}$ in $\mathfrak{C}$, those corresponding to transitive representations of $\Gamma$. We will pay particular attention to those categories for which the parent group $\Gamma$ is either an extended triangle group

$$
\Delta[p, q, r]=\left\langle R_{0}, R_{1}, R_{2} \mid R_{i}^{2}=\left(R_{1} R_{2}\right)^{p}=\left(R_{2} R_{0}\right)^{q}=\left(R_{0} R_{1}\right)^{r}=1\right\rangle,
$$

or its orientation-preserving subgroup of index 2 , the triangle group

$$
\Delta(p, q, r)=\left\langle X, Y, Z \mid X^{p}=Y^{q}=Z^{r}=X Y Z=1\right\rangle,
$$

where $X=R_{1} R_{2}, Y=R_{2} R_{0}$ and $Z=R_{0} R_{1}$. Here $p, q, r \in \mathbb{N} \cup\{\infty\}$, and we ignore any relations of the form $W^{\infty}=1$. We will now give some important examples of such 
categories; for more details, see [24]. In what follows, $C_{n}$ denotes a cyclic group of order $n, F_{n}$ denotes a free group of rank $n, V_{4}$ denotes a Klein four-group $C_{2} \times C_{2}$ and $*$ denotes a free product.

1. The category $\mathfrak{M}$ of all maps on surfaces (possibly non-orientable or with boundary) has parent group

$$
\Gamma=\Gamma_{\mathfrak{M}}=\Delta[\infty, 2, \infty] \cong V_{4} * C_{2} .
$$

This group acts on the set $\Omega$ of incident vertex-edge-face flags of a map (equivalently, the faces of its barycentric subdivision), with each generator $R_{i}(i=0,1,2)$ changing the $i$-dimensional component of each flag (whenever possible) while preserving the other two.

2. The subcategory $\mathfrak{M}^{+}$of $\mathfrak{M}$ consists of the oriented maps, those in which the underlying surface is oriented and without boundary. This category has parent group

$$
\Gamma=\Gamma_{\mathfrak{M}^{+}}=\Delta(\infty, 2, \infty) \cong C_{\infty} * C_{2},
$$

the orientation-preserving subgroup of index 2 in $\Delta[\infty, 2, \infty]$. This group acts on the directed edges of an oriented map: $X$ uses the local orientation to rotate them about their target vertices, and the involution $Y$ reverses their direction, so that $Z$ rotates them around incident faces. Here, and in the preceding example, $\Delta(p, 2, r)$ and $\Delta[p, 2, r]$ are the parent groups for the subcategories of maps of type $\{r, p\}$ in the notation of [10], meaning that the valencies of all vertices and faces divide $p$ and $r$ respectively, so that $X^{p}=Z^{r}=1$. (By convention, all positive integers divide $\infty$.)

3. Hypermaps are natural generalisation of maps, without the restriction that each edge is incident with at most two vertices and faces which implies that $Y^{2}=1$. There are several ways of defining or representing hypermaps. The most convenient way is via the Walsh bipartite map [54], where the black and white vertices correspond to the hypervertices and hyperedges of the hypermap, the edges correspond to incidences between them, and the faces correspond to its hyperfaces. The category $\mathfrak{H}$ of all hypermaps (possibly unoriented and with boundary) has parent group

$$
\Gamma=\Gamma_{\mathfrak{H}}=\Delta[\infty, \infty, \infty] \cong C_{2} * C_{2} * C_{2}
$$

This group acts on the incident edge-face pairs of the bipartite map, with $R_{0}$ and $R_{1}$ preserving the face and the incident white and black vertex respectively, while $R_{2}$ preserves the edge. As in the case of maps, $\Delta[p, q, r]$ is the parent group for the subcategory of hypermaps of type $(p, q, r)$.

4. For the subcategory $\mathfrak{H}^{+}$of oriented hypermaps, where the underlying surface is oriented and without boundary, the parent group is the even subgroup

$$
\Gamma=\Gamma_{\mathfrak{H}^{+}}=\Delta(\infty, \infty, \infty) \cong C_{\infty} * C_{\infty} \cong F_{2}
$$

of index 2 in $\Delta[\infty, 2, \infty]$. This acts on the edges of the bipartite map, with $X$ and $Y$ using the local orientation to rotate them around their incident black and white vertices, so that $Z$ rotates them around incident faces. Again $\Delta(p, q, r)$ is the parent group for the subcategory of oriented hypermaps of type $(p, q, r)$. Hypermaps of type $(p, 2, r)$ can be regarded as maps of type $\{r, p\}$ by deleting their white vertices; conversely maps correspond to hypermaps with $q=2$. 
5. One can regard the category $\mathfrak{D}$ of dessins d'enfants, introduced by Grothendieck [20], as the subcategory of $\mathfrak{H}^{+}$consisting of its finite objects, where the bipartite graph is finite and the surface is compact. The parent group is $\Gamma=\Delta(\infty, \infty, \infty) \cong F_{2}$, and its action is the same as for $\mathfrak{H}^{+}$.

Here we briefly mention two other classes of permutational categories where Theorem 1.1 applies.

6. Abstract polytopes [36] are higher-dimensional generalisations of maps. Those $n$ polytopes associated with the Schläfli symbol $\left\{p_{1}, \ldots, p_{n-1}\right\}$ can be regarded as transitive permutation representations of the string Coxeter group $\Gamma$ with presentation

$$
\left\langle R_{0}, \ldots, R_{n} \mid R_{i}^{2}=\left(R_{i-1} R_{i}\right)^{p_{i}}=\left(R_{i} R_{j}\right)^{2}=1(|i-j|>1)\right\rangle,
$$

acting on flags. For instance maps, in Example 1, correspond to the symbol $\{\infty, \infty\}$. However, in higher dimensions, not all transitive representations of $\Gamma$ correspond to abstract polytopes, since they need to satisfy the intersection property [36, Proposition 2B10].

7. Under suitable connectedness conditions (see [35, 39] for example), the connected, unbranched coverings $Y \rightarrow X$ of a topological space $X$ can be identified with the transitive permutation representations $\theta: \Gamma \rightarrow S=\operatorname{Sym}(\Omega)$ of its fundamental group $\Gamma=\pi_{1} X$, acting by unique path-lifting on the fibre $\Omega$ over a base-point in $X$. The automorphism group of an object $Y \rightarrow X$ in this category is its group of covering transformations, the centraliser in $S$ of the monodromy group $\theta(\Gamma)$ of the covering. For instance, dessins (see Example 5 above) correspond to finite unbranched coverings of the thrice-punctured sphere $X=\mathbb{P}^{1}(\mathbb{C}) \backslash\{0,1, \infty\}=\mathbb{C} \backslash\{0,1\}$, and hence to transitive finite permutation representations of its fundamental group $\Gamma=\pi_{1} X \cong F_{2} \cong \Delta(\infty, \infty, \infty)$. If we compactify surfaces by filling in punctures, then the unit interval $[0,1] \subset \mathbb{P}^{1}(\mathbb{C})$ lifts to a bipartite map on the covering surface $Y$, with black and white vertices over 0 and 1 , and face-centres over $\infty$. See $[17,29,31]$ for further details of these and other properties of dessins.

\section{Preliminary results}

In this section we will prove some general results which ensure that certain groups have various automorphism realisation properties.

Lemma 3.1. Let $\theta: \Gamma \rightarrow \Gamma^{\prime}$ be an epimorphism of groups. If $\Gamma^{\prime}$ is finitely or countably abundant or superabundant, then so is $\Gamma$.

Proof. If $A \cong N^{\prime} / M^{\prime}$ where $M^{\prime} \leq N^{\prime} \leq \Gamma^{\prime}$ and $N^{\prime}=N_{\Gamma^{\prime}}\left(M^{\prime}\right)$, then $A \cong N / M$ where $M=\theta^{-1}\left(M^{\prime}\right)$ and $N=\theta^{-1}\left(N^{\prime}\right)=N_{\Gamma}(M)$, with $|\Gamma: M|=\left|\Gamma^{\prime}: M^{\prime}\right|$, so $\Gamma$ inherits finite or countable abundance from $\Gamma^{\prime}$. Moreover, non-conjugate subgroups $M^{\prime}$ lift to non-conjugate subgroups $M$, so the superabundance properties are also inherited.

Our basic tool for proving finite superabundance will be the following:

Proposition 3.2. Let $\Gamma$ be a group with a sequence $\left\{N_{n} \mid n \geq n_{0}\right\}$ of maximal subgroups $N_{n}$ of finite index such that for each $a, d \in \mathbb{N}$ there is some $n$ with $\left|N_{n}: K_{n}\right|>a$, where $K_{n}$ is the core of $N_{n}$ in $\Gamma$, and there is an epimorphism $N_{n} \rightarrow F_{d}$. Then $\Gamma$ is finitely superabundant. 
Proof. Any finite group $A$ is an $d$-generator group for some $d \in \mathbb{N}$, so there is an epimorphism $F_{d} \rightarrow A$. By hypothesis, for some maximal subgroup $N=N_{n}$ of $\Gamma$ there is an epimorphism $N \rightarrow F_{d}$, and the core $K$ of $N$ satisfies $|N: K|>|A|$. Composition gives an epimorphism $N \rightarrow A$, and hence a normal subgroup $M$ of $N$ with $N / M \cong A$. Then $N_{\Gamma}(M) \geq N$, so the maximality of $N$ implies that either $N=N_{\Gamma}(M)$ or $M$ is a normal subgroup of $\Gamma$. If $M$ is normal in $\Gamma$ then $M$ must be contained in the core $K$ of $N$, so that $|N: M| \geq|N: K|$. But this is impossible, since $|N: M|=|A|$ and we chose $N=N_{n}$ so that $|N: K|>|A|$. Hence $N=N_{\Gamma}(M)$, as required. Moreover, given $A$ we can find such subgroups $N$ with $|N: K|$ arbitrarily large, so infinitely many of them are mutually non-conjugate, and hence so are their corresponding subgroups $M$, since conjugate subgroups have conjugate normalisers.

In order to deal with countable abundance or superabundance we need an analogue of Proposition 3.2 for countable groups $A$. Here we have the advantage that, instead of an infinite sequence of maximal subgroups, which are finitely generated if $\Gamma$ is, a single infinitely generated maximal subgroup is sufficient. However, when $A$ is infinite we cannot ensure that $M$ is not normal in $\Gamma$ simply by comparing indices of subgroups, since these are not finite; a new idea is therefore needed.

Proposition 3.3. Let $\Gamma$ be a group with a non-normal maximal subgroup $N$ and an epimorphism $\phi: N \rightarrow F_{\infty}$. Then $\Gamma$ is countably abundant. Moreover, each countable group $A \neq 1$ is realised as $N_{\Gamma}(M) / M$ by $2^{\aleph_{0}}$ conjugacy classes of subgroups $M$ in $\Gamma$ with $N_{\Gamma}(M)=N$.

Proof. Given any countable group $A$ there exist epimorphisms $\alpha: F_{\infty} \rightarrow A$; composing any of these with the epimorphism $\phi: N \rightarrow F_{\infty}$ gives an epimorphism $\phi \circ \alpha: N \rightarrow A$, and hence a normal subgroup $M=\operatorname{ker}(\phi \circ \alpha)$ of $N$ with $N / M \cong A$. As before, the maximality of $N$ implies that either $N=N_{\Gamma}(M)$, as required, or $M$ is a normal subgroup of $\Gamma$. In the latter case $M$ is contained in the core $K$ of $N$ in $\Gamma$, so to prove the result we need to show that we can choose $\alpha$ so that $M \not \subset K$. Since $N$ is not normal in $\Gamma$ we have $N \backslash K \neq \emptyset$, so choose any element $g \in N \backslash K$, and define $f:=g \phi \in F_{\infty}$. Then we can choose $\alpha: F_{\infty} \rightarrow A$ so that all of the (finitely many) free generators of $F_{\infty}$ appearing in $f$ are in $\operatorname{ker}(\alpha)$, and hence $g \in M$. Thus $M \not \subset K$, so $\Gamma$ is countably abundant.

If $A \neq 1$ we can choose such epimorphisms $\alpha$ with $2^{\aleph_{0}}$ different kernels, lifting back to distinct subgroups $M$ of $\Gamma$; these all have normaliser $N$, which is its own normaliser in $\Gamma$, so they are mutually non-conjugate in $\Gamma$, giving us $2^{\aleph_{0}}$ conjugacy classes of subgroups $M$ realising $A$.

Remark 3.4. Unfortunately, if $A=1$ then $\alpha$ is unique, so that $M=N$, and the subgroup $N$ yields only one conjugacy class of subgroups realising $A$. In this case, in order to prove that $\Gamma$ is countably superabundant by this construction we would need to find not one but $2^{\aleph_{0}}$ conjugacy classes of non-normal maximal subgroups $N$. For certain specific groups $\Gamma$ we will be able to do this.

\section{Finite superabundance of hyperbolic triangle and extended triangle groups}

In this section we will use Proposition 3.2 to prove Theorem 1.3(a). 
Case 1: $\Gamma=\Delta(p, q, r)$, cocompact. First assume that $\Gamma=\Delta(p, q, r)$, acting cocompactly on the hyperbolic plane, that is, with finite periods $p, q$ and $r$. By Dirichlet's Theorem on primes in an arithmetic progression, there are infinitely many primes $n \equiv-1 \bmod (l)$, where $l:=\operatorname{lcm}\{2 p, 2 q, 2 r\}$. For each such $n$ there is an epimorphism $\theta_{n}: \Gamma \rightarrow \operatorname{PSL}_{2}(n)$ sending the standard generators $X, Y$ and $Z$ of $\Gamma$ to elements $x, y$ and $z$ of $\operatorname{PSL}_{2}(n)$ of orders $p, q$ and $r$ (see [16, Corollary C], for example). This gives an action of $\Gamma$ on the projective line $\mathbb{P}^{1}\left(\mathbb{F}_{n}\right)$, which is doubly transitive and hence primitive, so the subgroup $N_{n}$ of $\Gamma$ fixing $\infty$ is a non-normal maximal subgroup of index $n+1$. Since $p, q$ and $r$ all divide $(n+1) / 2$, the elements $x, y$ and $z$ are semi-regular permutations on $\mathbb{P}^{1}\left(\mathbb{F}_{n}\right)$, with all their cycles of length $p, q$ or $r$. Thus no non-identity powers of $X, Y$ or $Z$ have fixed points, so by a theorem of Singerman [48] $N_{n}$ is a surface group

$$
N_{n}=\left\langle A_{i}, B_{i}(i=1, \ldots, g) \mid \prod_{i=1}^{g}\left[A_{i}, B_{i}\right]=1\right\rangle
$$

of genus $g$ given by the Riemann-Hurwitz formula:

$$
2(g-1)=(n+1)\left(1-\frac{1}{p}-\frac{1}{q}-\frac{1}{r}\right) .
$$

This shows that $g \rightarrow \infty$ as $n \rightarrow \infty$. Now we can map $N_{n}$ onto the free group $F_{g}$ by sending the generators $A_{i}$ to a free basis, and the generators $B_{i}$ to 1 . The core $K_{n}=\operatorname{ker}\left(\theta_{n}\right)$ of $N_{n}$ in $\Gamma$ satisfies $\left|N_{n}: K_{n}\right|=\left|\mathrm{PSL}_{2}(n)\right| /(n+1)=n(n-1) / 2$, so Proposition 3.2 gives the result.

Case 2: $\Gamma=\Delta(p, q, r)$, not cocompact. Now assume that $\Gamma$ has $k$ infinite periods $p, q, r$ for some $k=1,2$ or 3 . We can adapt the above argument by first choosing an infinite set of primes $n \geq 13$ such that any finite periods of $\Gamma$ divide $(n+1) / 2$, as before. For each such $n$ we can map $\Gamma$ onto a cocompact triangle group $\Gamma_{n}$, where each infinite period of $\Gamma$ is replaced with $(n+1) / 2$. Since $(n+1) / 2 \geq 7$, the triangle group $\Gamma_{n}$ is also hyperbolic, so as before there is an epimorphism $\Gamma_{n} \rightarrow \mathrm{PSL}_{2}(n)$, giving (by composition) a primitive action of $\Gamma$ on $\mathbb{P}^{1}\left(\mathbb{F}_{n}\right)$. Again, no non-identity powers of any elliptic generators among $X, Y$ and $Z$ have fixed points, but any parabolic generator (one of infinite order) now induces two cycles of length $(n+1) / 2$ on $\mathbb{P}^{1}\left(\mathbb{F}_{n}\right)$, so by [48] it introduces two parabolic generators $P_{i}$ into the standard presentation of the point-stabiliser $N_{n}$ in $\Gamma$. We therefore have

$$
N_{n}=\left\langle A_{i}, B_{i}(i=1, \ldots, g), P_{i}(i=1, \ldots, 2 k) \mid \prod_{i=1}^{g}\left[A_{i}, B_{i}\right] . \prod_{i=1}^{2 k} P_{i}=1\right\rangle,
$$

a free group of rank $2 g+2 k-1$, where the Riemann-Hurwitz formula now gives

$$
2(g-1)+2 k=(n+1)\left(1-\frac{1}{p}-\frac{1}{q}-\frac{1}{r}\right)
$$

with $1 / \infty=0$. Since $k \leq 3$ we have $g \rightarrow \infty$ as $n \rightarrow \infty$, so Proposition 3.2 again gives the result.

Case 3: $\Gamma=\Delta[p, q, r]$. The proof when $\Gamma$ is an extended triangle group $\Delta[p, q, r]$ of hyperbolic type is similar to that for $\Delta(p, q, r)$. If $\Gamma$ is cocompact then, as before, we 
consider epimorphisms $\theta_{n}: \Gamma^{+}=\Delta(p, q, r) \rightarrow \mathrm{PSL}_{2}(n)$ for primes $n \equiv-1 \bmod (l)$, where now $l=\operatorname{lcm}\{2 p, 2 q, 2 r, 4\}$; the stabilisers of $\infty$ form a series of maximal subgroups $N_{n}$ of index $n+1$ in $\Gamma^{+}$. By an observation of Singerman [50] the core $K_{n}$ of $N_{n}$ in $\Gamma^{+}$is normal in $\Gamma$, with quotient $\Gamma / K_{n}$ isomorphic to $\mathrm{PSL}_{2}(n) \times C_{2}$ or $\mathrm{PGL}_{2}(n)$ as the automorphism of $\operatorname{PSL}_{2}(n)$ inverting $x$ and $y$ is inner or not. Thus $\theta_{n}$ extends to a homomorphism $\theta_{n}^{*}: \Gamma \rightarrow \mathrm{PGL}_{2}(n)$; in the first case its image is $\mathrm{PSL}_{2}(n)$ and its kernel $K_{n}^{*}$ contains $K_{n}$ with index 2 , and in the second case it is an epimorphism with kernel $K_{n}^{*}=K_{n}$. In either case the action of $\Gamma^{+}$on $\mathbb{P}^{1}\left(\mathbb{F}_{n}\right)$ extends to an action of $\Gamma$, and the stabiliser $N_{n}^{*}$ of $\infty$ is a maximal subgroup of index $n+1$ in $\Gamma$, containing $N_{n}$ with index 2. In order to apply Proposition 3.2 to these subgroups $N_{n}^{*}$ it is sufficient to show that they map onto free groups of unbounded rank.

Now $N_{n}^{*}$ is a non-euclidean crystallographic (NEC) group, and $N_{n}$ is its canonical Fuchsian subgroup of index 2. We can obtain the signature of $N_{n}^{*}$ by using Hoare's extension to NEC groups [22] of Singerman's results [48] on subgroups of Fuchsian groups. As before, $N_{n}$ is a surface group of genus $g$ given by (4.1). There are no elliptic or parabolic elements in $N_{n}$, and hence none in $N_{n}^{*}$. The reflections $R_{i}(i=0,1,2)$ generating $\Gamma$ induce involutions on $\mathbb{P}^{1}\left(\mathbb{F}_{n}\right)$, each with at most two fixed points. If $\Gamma / K_{n} \cong \operatorname{PSL}_{2}(n) \times C_{2}$ these involutions are elements of $\mathrm{PSL}_{2}(n)$, so they are even permutations by the simplicity of this group, and hence they have no fixed points since $n+1 \equiv 0 \bmod (4)$. Thus $N_{n}^{*}$ contains no reflections; however, it is not a subgroup of $\Gamma^{+}$, so it is a non-orientable surface group

$$
N_{n}^{*}=\left\langle G_{1}, \ldots, G_{g^{*}} \mid G_{1}^{2} \ldots G_{g^{*}}^{2}=1\right\rangle
$$

generated by glide-reflections $G_{i}$, with its genus $g^{*}$ given by the Riemann-Hurwitz formula

$$
2-2 g=2\left(2-g^{*}\right)
$$

for the inclusion $N_{n} \leq N_{n}^{*}$, so $g^{*}=g+1$. Thus there is an epimorphism $N_{n}^{*} \rightarrow F_{d}=$ $\left\langle X_{1}, \ldots, X_{d} \mid-\right\rangle$ where $d=\left\lfloor g^{*} / 2\right\rfloor$, given by $G_{2 i-1} \mapsto X_{i}$ and $G_{2 i} \mapsto X_{i}^{-1}$ for $i=1, \ldots, d$ and $G_{g^{*}} \mapsto 1$ if $g^{*}$ is odd. Since $g \rightarrow \infty$ as $n \rightarrow \infty$, we have $d \sim g / 2 \rightarrow \infty$ also, so Proposition 3.2 gives the result.

Similar arguments also deal with the case where $\Gamma / K_{n} \cong \mathrm{PGL}_{2}(n)$. Since $n \equiv-1$ mod (4), each generating reflection $R_{i}$ of $\Gamma$ induces an odd permutation of $\mathbb{P}^{1}\left(\mathbb{F}_{n}\right)$ with two fixed points, contributing two reflections to the standard presentation of the NEC group $N_{n}^{*}$. The Riemann-Hurwitz formula for the inclusion $N_{n} \leq N_{n}^{*}$ then takes the form

$$
2-2 g=2\left(2-h^{*}+s\right),
$$

where $h^{*}=2 g^{*}$ or $g^{*}$ as $N_{n}^{*}$ has an orientable or non-orientable quotient surface of genus $g^{*}$ with $s$ boundary components for some $s \leq 6$. Thus $h^{*} \sim g \rightarrow \infty$ as $n \rightarrow \infty$. We obtain an epimorphism $N_{n}^{*} \rightarrow F_{d}$ with $d \sim h^{*} / 2$ as in the orientable or non-orientable cases above, this time by mapping the additional standard generators of $N_{n}^{*}$, associated with the boundary components, to 1 , so Proposition 3.2 again gives the result. Finally, in the non-cocompact case, any periods $p, q, r=\infty$ can be dealt with as above for $\Delta(p, q, r)$. This completes the proof of Theorem 1.3(a).

Remark 4.1. It seems plausible that an argument based on the Čebotarev Density Theorem would show that, given $\Gamma=\Delta[p, q, r]$, the cases $\Gamma / K_{n} \cong \mathrm{PSL}_{2}(n) \times C_{2}$ and $\mathrm{PGL}_{2}(n)$ each occur for infinitely many primes $n \equiv-1 \bmod (l)$, so that only one case would need to 
be considered; however, the resulting shortening of the proof would not justify the effort. Nevertheless this dichotomy, for general prime powers $n$, is interesting in its own right and deserves further study.

Remark 4.2. The restrictions on the prime $n$ in the above proof are partly for convenience of exposition, rather than necessity. Relaxing them would allow $X, Y$ and $Z$ to have one or two fixed points on $\mathbb{P}^{1}\left(\mathbb{F}_{n}\right)$, thus adding extra standard generators to $N_{n}$ and $N_{n}^{*}$ and extra summands to the Riemann-Hurwitz formulae used. However, these extra terms are bounded as $n \rightarrow \infty$, so asymptotically they make no significant difference. One advantage of these restrictions is that since $x, y$ and $z$ are semi-regular permutations, the hypermaps realising $A$ in Case 1 are uniform, that is, their hypervertices, hyperedges and hyperfaces all have valencies $p, q$ and $r$. If we choose these periods so that $\Delta(p, q, r)$ is cocompact, maximal (see [49]) and non-arithmetic (see [52]), then by a result of Singerman and Syddall [51, Theorem 12.1] each hypermap (regarded as a dessin) has the same automorphism group as its underlying Riemann surface. By [49, 52] these conditions apply to 'most' hyperbolic triples, such as $(2,3,13)$, so we have the following:

Corollary 4.3. The category of compact Riemann surfaces is finitely superabundant.

In fact, Greenberg [19, Theorem 6'] showed in 1973 that, given a compact Riemann surface $S$ and a finite group $A \neq 1$, there is a normal covering $T \rightarrow S$ with covering group and $\operatorname{Aut}(T)$ both isomorphic to $A$, while Teichmüller theory yields uncountably many compact Riemann surfaces realising $A$. Since the Riemann surfaces realising a finite group $A$ in Corollary 4.3 are uniformised by subgroups of finite index in triangle groups, by Grothendieck's reinterpretation [20] of Belyı̆'s Theorem they are all defined (as algebraic curves with automorphism group $A$ ) over number fields.

\section{Countable abundance of non-cocompact hyperbolic triangle groups}

We now turn to Theorem 1.3(b) and consider countable abundance, starting with the hyperbolic triangle groups $\Gamma=\Delta(p, q, r)$. We would like to show that $\Gamma$ satisfies the hypotheses of Proposition 3.3, that is, it has a non-normal maximal subgroup which has an epimorphism onto $F_{\infty}$. Given $\Gamma$, it is easy to find maximal subgroups of finite index by mapping $\Gamma$ onto primitive permutation groups of finite degree; however, such subgroups are finitely generated, so they do not map onto $F_{\infty}$; a maximal subgroup of infinite index is needed, and these seem to be harder to find. They certainly exist: by a result of Ol'shanskir [43], $\Gamma$ has a quotient $Q \neq 1$ with no proper subgroups of finite index; by Zorn's Lemma, $Q$ has maximal subgroups, which must have infinite index, and these lift back to maximal subgroups of infinite index in $\Gamma$. These are not normal (otherwise they would have prime index), but does one of them map onto $F_{\infty}$ ? Conceivably, they could be generated by elliptic elements, which have finite order, in which case they would not map onto a free group of any rank. As a first step we consider the case where $\Gamma$ is not cocompact, that is, it has an infinite period, so it is a free product of two cyclic groups. For simplicity of exposition we first consider countable abundance, postponing superabundance until the next section.

Theorem 5.1. If $\Gamma$ is a non-cocompact hyperbolic triangle group $\Delta(p, q, r)$, then $\Gamma$ and the corresponding category of oriented hypermaps are countably abundant.

Proof. By Proposition 3.3 it is sufficient to show that $\Gamma$ has a non-normal maximal subgroup $N$ with an epimorphism $N \rightarrow F_{\infty}$. Using the usual isomorphisms between triangle 
groups, we may assume that $r=\infty$, and that $p \geq 3$ and $q \geq 2$, so that $\Gamma \cong C_{p} * C_{q}$ with $p, q \in \mathbb{N} \cup\{\infty\}$.

Case 1: $p=3, q=2$. First we consider the case where $p=3$ and $q=2$, so that $\Gamma$ is isomorphic to the modular group $\mathrm{PSL}_{2}(\mathbb{Z}) \cong C_{3} * C_{2}$. We can construct a maximal subgroup $N$ of infinite index in $\Gamma$ as the point stabiliser in a primitive permutation representation of $\Gamma$ of infinite degree. Since $\Gamma$ is the parent group

$$
\Delta(3,2, \infty)=\left\langle X, Y, Z \mid X^{3}=Y^{2}=X Y Z=1\right\rangle
$$

for the category $\mathfrak{C}=\mathfrak{M}_{3}^{+}$of oriented trivalent maps, we can take $N$ to be the subgroup of $\Gamma$ corresponding to an infinite map $\mathcal{N}_{3}$ in $\mathfrak{C}$.

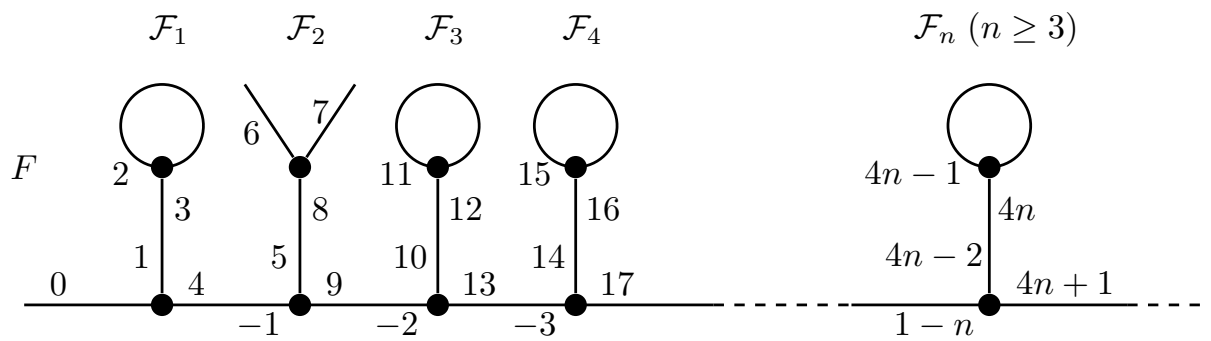

Figure 1: The trivalent map $\mathcal{N}_{3}$.

We will take $\mathcal{N}_{3}$ to be the infinite planar trivalent map shown in Figure 1, oriented with the positive (anticlockwise) orientation of the plane. The monodromy group $G=\langle x, y\rangle$ of this map gives a transitive permutation representation $\theta: \Gamma \rightarrow G, X \mapsto x, Y \mapsto y, Z \mapsto z$ of $\Gamma$ on the set $\Omega$ of directed edges of $\mathcal{N}_{3}$, with $x$ rotating them anticlockwise around their target vertices, and $y$ reversing their direction. The vertices, all of valency 3 , correspond to the 3-cycles of $x$ (it has no fixed points). The edges correspond to the cycles of $y$, with three free edges corresponding to its fixed points and the other edges corresponding to its 2 -cycles. The faces correspond to the cycles of $z=y x^{-1}$, and in particular, the directed edge $\alpha$ labelled 0 and fixed by $y$ is in an infinite cycle $C=\left(\ldots, \alpha z^{-1}, \alpha, \alpha z, \ldots\right)$ of $z$, corresponding to the unbounded face $F$ of $\mathcal{N}_{3}$; the directed edges $\alpha z^{i}$ in $C$ are indicated by integers $i$ in Figure 1. The unlabelled directed edges are fixed points of $z$, one incident with each 1-valent face. The pattern seen in Figure 1 repeats to the right in the obvious way. The 'flowers' $\mathcal{F}_{n}(n \geq 1)$ above the horizontal axis continue indefinitely to the right, with $\mathcal{F}_{n}$ an identical copy of $\mathcal{F}_{1}$ for each $n \geq 3$; we will later need the fact that for each $n \geq 2$ the 'stem' of $\mathcal{F}_{n}$ (the vertical edge connecting it to the horizontal axis) carries two directed edges in $C$, with only one of their two labels divisible by $n$.

Lemma 5.2. The group $\Gamma$ acts primitively on $\Omega$.

Proof. Suppose that $\sim$ is a $\Gamma$-invariant equivalence relation on $\Omega$; we need to show that it is either the identity or the universal relation. Since $\alpha y=\alpha$, the equivalence class $E=[\alpha]$ containing $\alpha$ satisfies $E y=E$. Since $\langle Z\rangle$ acts regularly on $C$ we can identify $C$ with $\mathbb{Z}$ by identifying each $\alpha z^{i} \in C$ with the integer $i$, so that $Z$ acts by $i \mapsto i+1$. Then $\sim$ restricts to a translation-invariant equivalence relation on $\mathbb{Z}$, which must be congruence $\bmod (n)$ for 
some $n \in \mathbb{N} \cup\{\infty\}$, where we include $n=1$ and $\infty$ for the universal and identity relations on $\mathbb{Z}$.

Suppose first that $n \in \mathbb{N}$, so $E \cap C$ is the subgroup $(n)$ of $\mathbb{Z}$. If $n=1$ then $C \subseteq E$. Now $E x^{-1}$ is an equivalence class, and it contains $\alpha x^{-1}=1$; this is in $C$, and hence in $E$, so $E x^{-1}=E$. We have seen that $E y=E$, so $E=\Omega$ since $G=\left\langle x^{-1}, y\right\rangle$, and hence $\sim$ is the universal relation on $\Omega$.

We may therefore assume that $n>1$. The vertical stem of the flower $\mathcal{F}_{n}$ is an edge carrying two directed edges in $C$, with only one of its two labels divisible by $n$, so one is in $E$ whereas the other is not. However, these two directed edges are transposed by $y$, contradicting the fact that $E y=E$.

Finally suppose that $n=\infty$, so that all elements of $C$ are in distinct equivalence classes, and hence the same applies to $C y$. In particular, since $\alpha \in C \cap C y$ we have $E \cap C=\{\alpha\}=E \cap C y$. By inspection of Figure 1, $\Omega=C \cup C y$ and hence $E=\{\alpha\}$. It follows that all equivalence classes for $\sim$ are singletons, so $\sim$ is the identity relation, as required.

We now return to the proof of Theorem 5.1. It follows from Lemma 5.2 that the subgroup $N=\Gamma_{\alpha}$ of $\Gamma$ fixing $\alpha$ is maximal. Clearly $N$ is not normal in $\Gamma$, since $G$ is not a regular permutation group, so it sufficient to find an epimorphism $N \rightarrow F_{\infty}$. One could use the Reidemeister-Schreier algorithm to find a presentation for $N$ : truncation converts $\mathcal{N}_{3}$ into a coset diagram for $N$ in $\Gamma$, and then deleting edges to form a spanning tree yields a Schreier transversal. In fact a glance at Figure 1 shows that $N$ is a free product of cyclic groups: three of these, corresponding to the fixed points of $y$ and generated by conjugates of $Y$, have order 2 , and there are infinitely many of infinite order, generated by conjugates of $Z$ and corresponding to the fixed points of $z$, that is, the 1 -valent faces of $\mathcal{N}_{3}$, one in each flower $\mathcal{F}_{n}$ for $n \neq 2$. By mapping the generators of finite order to the identity we obtain the required epimorphism $N \rightarrow F_{\infty}$.

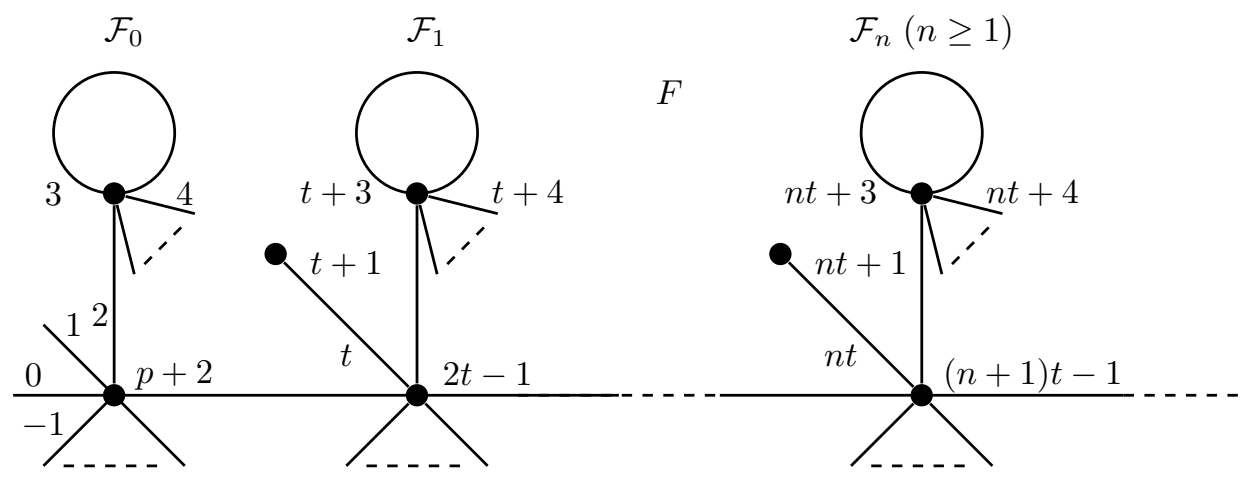

Figure 2: The $p$-valent $\operatorname{map} \mathcal{N}_{p}$, with $t:=p+3$.

Case 2: Finite $p \geq 4, q=2$. We now assume that $\Gamma$ is a Hecke group $C_{p} * C_{2}$ for some finite $p \geq 4$. Let $\mathcal{N}_{p}$ be the infinite $p$-valent planar map in Figure 2. Apart from $\mathcal{F}_{0}$, the flowers are all identical copies of $\mathcal{F}_{1}$, with a 'leaf' growing out of its base and leading to a vertex of valency 1 , representing a fixed point of $x$. The 'fans' indicated by short dashed lines represent however many free edges are needed in order that the incident vertex should have valency $p$, that is, $p-3$ free edges for vertices at the top of a stem, and $p-4$ for 
those at the base. As before, the elements $\alpha z^{i}$ of the cycle $C$ of $z$ containing the directed edge $\alpha=0$ are labelled with integers $i$; to save space in the diagram only a few labels are shown. We define $t=p+3$, since a translation from a flower $\mathcal{F}_{n}(n \geq 1)$ to the next flower $\mathcal{F}_{n+1}$ adds that number to all labels.

The proof that the monodromy group $G=\langle x, y\rangle$ of $\mathcal{N}_{p}$ is primitive is very similar to that in Lemma 5.2 for $\mathcal{N}_{3}$. Any $\Gamma$-invariant equivalence relation $\sim$ on $\Omega$ restricts to $C$ as congruence $\bmod (n)$ for some $n \in \mathbb{N} \cup\{\infty\}$. The equivalence class $E=[\alpha]$ satisfies $E y=E$, so if $n \neq 1, \infty$ then the fact that $y$ transposes the directed edges labelled $n t$ and $n t+1$, with the first but not the second in $E=(n)$, gives a contradiction. If $n=1$ then $C \subseteq E$, so both $x$ and $y$ preserve $E$ and hence $\sim$ is the universal relation. If $n=\infty$ then $E \cap C=\{\alpha\}$, and hence also $E \cap C y=\{\alpha\}$; but $\Omega=C \cup C y$ and hence $E=\{\alpha\}$ and $\sim$ is the identity relation.

This shows that the subgroup $N$ of $\Gamma$ fixing $\alpha$ is maximal. As before, it is not normal, and it is a free product of cyclic groups, now of order $p, 2$ or $\infty$, corresponding to the fixed points of $x, y$ and $z$ (infinitely many in each case). Sending the generators of finite order to the identity gives the required epimorphism $N \rightarrow F_{\infty}$.

Case 3: Finite $p, q \geq 3$. We modify the map $\mathcal{N}_{p}$ in the proof of Case 2 by removing the leaf attached to the base of each flower $\mathcal{F}_{n}(n \geq 1)$, adding a white vertex to every remaining edge (including one at the free end of each free edge), and finally adding edges incident with 1 -valent black vertices where necessary to ensure that all white vertices have valency $q$ or 1 . The resulting map $\mathcal{N}_{p, q}$ is shown in Figure 3.

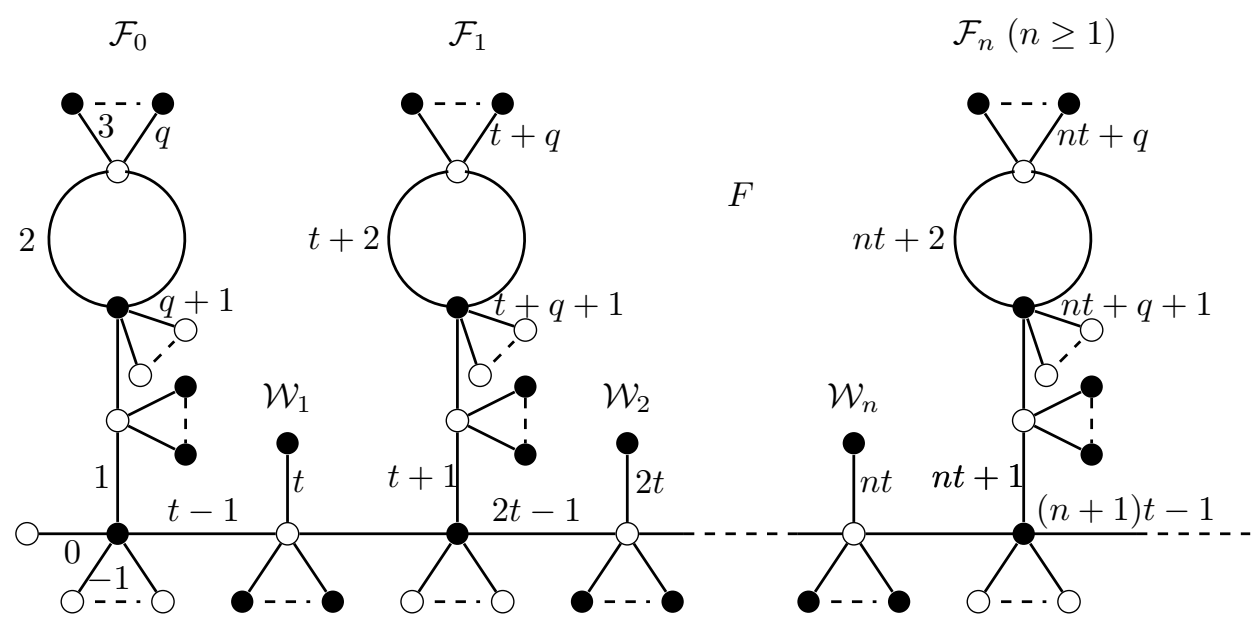

Figure 3: The bipartite map $\mathcal{N}_{p, q}$, with $t:=p+2 q-2$.

Note that while the flowers $\mathcal{F}_{n}$ have grown since Case 2 was proved, small 'weeds' $\mathcal{W}_{n}(n \geq 1)$ have grown between them. This bipartite map is the Walsh map for an oriented hypermap of type $(p, q, \infty)$. Its monodromy group $G$ is generated by permutations $x$ and $y$, of order $p$ and $q$, which rotate edges around their incident black and white vertices. It is sufficient to show that $G$ acts primitively on the set $\Omega$ of edges, and that in the induced action of $\Gamma$ on $\Omega$, the subgroup $N$ fixing an edge has an epimorphism onto $F_{\infty}$.

The proof is similar to that for Case 2. The elements $\alpha z^{i}$ of the cycle $C$ of $z$ containing 
the edge $\alpha=0$ are labelled with integers $i$. (To save space in Figure 3, only a few significant labels are shown.) Any $\Gamma$-invariant equivalence relation $\sim$ restricts to $C$ as congruence $\bmod (n)$ for some $n \in \mathbb{N} \cup\{\infty\}$. If $E=[\alpha]$ then since $\alpha y=\alpha$ we have $E y=E$. If $n \neq \infty$ then $E x=E$ since the edge $\beta \in E$ labelled $n t$ is fixed by $x$, so that $E \Gamma=E$ and hence $E=\Omega$. Thus we may assume that $n=\infty$, so all elements of $C$ are in distinct conjugacy classes and hence $E \cap C=\{\alpha\}$. Similarly $E \cap C y=\{\alpha\}$. But $\Omega=C \cup C y$, so $E=\{\alpha\}$ and $\sim$ is the identity relation. Thus $G$ is primitive, so the subgroup $N$ of $\Gamma$ fixing $\alpha$ is maximal. It is a free product of cyclic groups, of orders $p, q$ and $\infty$, corresponding to the fixed points of $x, y$ and $z$. There are infinitely many of each, and mapping those of finite order to the identity gives an epimorphism $N \rightarrow F_{\infty}$.

Case 4: $p$ or $q=\infty$. If $p=\infty$ or $q=\infty$ we can use the natural epimorphism from $\Gamma=\Delta(p, q, \infty)$ to a hyperbolic triangle group $\Gamma^{\prime}=\Delta\left(p^{\prime}, q^{\prime}, \infty\right)$ with $p^{\prime}$ and $q^{\prime}$ both finite, use Case 1, 2 or 3 to establish countable abundance for $\Gamma^{\prime}$, and finally use Lemma 3.1 to deduce it for $\Gamma$.

Remark 5.3. Constructions similar to those in the proof of Theorem 5.1 have been used in [25] to prove that if $\Gamma$ is a non-cocompact hyperbolic triangle group then $\Gamma$ has uncountably many conjugacy classes of maximal subgroups of infinite index. This strengthens and generalises results of B. H. Neumann [40], Magnus [33, 34], Tretkoff [53], and Brenner and Lyndon [6] on maximal nonparabolic subgroups of the modular group, and has some overlap with work of Kulkarni [30] on maximal subgroups of free products of cyclic groups.

\section{Countable superabundance of non-cocompact hyperbolic triangle groups}

In order to prove countable superabundance for non-cocompact hyperbolic triangle groups $\Gamma=\Delta(p, q, r)$, we need $2^{\aleph_{0}}$ objects realising each countable group $A$. The proofs of countable abundance for the various cases in Theorem 5.1 all used Proposition 3.3, and by Remark 3.4 this yields the required number of objects in all cases except when $A \neq 1$.

In fact, for any countable group $A$ these proofs can be adapted (as in Remark 5.3) to produce not just one but $2^{\aleph_{0}}$ conjugacy classes of subgroups $N$ satisfying the conditions of Proposition 3.3. We thus obtain $2^{\aleph_{0}}$ non-isomorphic objects $\mathcal{N}$, each with $2^{\aleph_{0}}$ coverings $\mathcal{O}$ realising any countable group $A \neq 1$, and with one covering (namely $\mathcal{O}=\mathcal{N}$ ) realising $A=1$; each $\mathcal{O}$ has the property that $\mathcal{O} / \operatorname{Aut}(\mathcal{O}) \cong \mathcal{N}$, so $A$ is realised by $2^{\aleph_{0}}$ nonisomorphic objects.

We will give the required details for Case 1 of Theorem 5.1, where $p=3, q=2$ and $\Gamma$ is the modular group; the argument is similar in the other cases. We can modify the map $\mathcal{N}_{3}$ in Figure 1 by adding 'stalks' between the flowers $\mathcal{F}_{n}$, each consisting of a new vertex on the horizontal axis, and a new free edge pointing upwards. Adding a stalk between $\mathcal{F}_{m}$ and $\mathcal{F}_{m+1}$ adds 2 to the value of all labels on flowers $\mathcal{F}_{n}$ for $n>m$. For the proof of Theorem 5.1 to work we need to preserve the property that only one of the two labels on the stem of each flower $\mathcal{F}_{n}(n>1)$ is divisible by $n$. This can be done, in $2^{\aleph_{0}}$ different ways, by ensuring that for each $n>1$ the total number of stalks added between $\mathcal{F}_{1}$ and $\mathcal{F}_{n}$ is a multiple of $n$. The proof for Case 1 then proceeds as before, except that it now yields $2^{\aleph_{0}}$ conjugacy classes of maximal subgroups $N$.

In the remaining cases of Theorem 5.1 we could use similar modifications to the maps 
$\mathcal{N}_{p}$ and $\mathcal{N}_{p, q}$ in Figures 2 and 3, or alternatively add extra vertices and edges to those below the horizontal axis, so that the non-negative labels above the axis are unaltered.

\section{Countable superabundance of non-cocompact extended triangle groups}

We now consider countable superabundance for extended triangle groups $\Gamma=\Delta[p, q, r]$ and their associated categories of unoriented hypermaps, again restricting attention to noncocompact groups. Earlier we realised countable groups $A$ as automorphism groups in various categories $\mathfrak{C}^{+}$of oriented hypermaps of a given type by constructing specific objects $\mathcal{N}=\mathcal{N}_{p}(p \geq 3)$ or $\mathcal{N}_{p, q}(p, q \geq 3)$ in those categories, and then forming regular coverings $\mathcal{M}$ of $\mathcal{N}$, with covering group $A$, constructed so that $\mathcal{M}$ has only those automorphisms induced by $A$. These objects $\mathcal{M}$ and $\mathcal{N}$ correspond to subgroups $M$ and $N$ of the parent group $\Gamma^{+}=\Delta(p, q, r)$ for $\mathfrak{C}^{+}$with $N=N_{\Gamma^{+}}(M)$. We can also regard $\mathcal{M}$ and $\mathcal{N}$ as objects in the corresponding category $\mathfrak{C}$ of unoriented maps or hypermaps of type $(p, q, r)$, for which the parent group is the extended triangle group $\Gamma$.

Lemma 7.1. For these objects $\mathcal{M}$ we have $\operatorname{Aut}_{\mathfrak{C}}(\mathcal{M})=\operatorname{Aut}_{\mathfrak{C}^{+}}(\mathcal{M}) \cong A$.

Proof. We have $\operatorname{Aut}_{\mathfrak{C}}(\mathcal{M}) \cong N_{\Gamma}(M) / M$ and $\operatorname{Aut}_{\mathfrak{C}^{+}}(\mathcal{M}) \cong N_{\Gamma^{+}}(M) / M \cong A$ by Theorem 1.1, so it is sufficient to show that $N_{\Gamma}(M)=N_{\Gamma^{+}}(M)$. Clearly $N_{\Gamma}(M) \geq N_{\Gamma^{+}}(M)$. If this inclusion is proper then since $N_{\Gamma^{+}}(M)=N_{\Gamma}(M) \cap \Gamma^{+}$with $\left|\Gamma: \Gamma^{+}\right|=2$ we have $\left|N_{\Gamma}(M): N_{\Gamma^{+}}(M)\right|=2$, so the subgroup $N=N_{\Gamma^{+}}(M)$ is normalised by some elements of $\Gamma \backslash \Gamma^{+}$. This is impossible, since in all cases the map or hypermap $\mathcal{N}$ corresponding to $N$ is chiral (without orientation-reversing automorphisms), by the proof of Theorem 5.1 and by inspection of Figures 1,2 and 3. The same applies to the modified maps required to produce $2^{\aleph_{0}}$ such objects $\mathcal{O}$.

Corollary 7.2. Each non-cocompact hyperbolic extended triangle group $\Delta[p, q, r]$ and its associated category of all hypermaps of type $(p, q, r)$ are countably superabundant.

This completes the proof of Theorem 1.3(b).

Remark 7.3. It would not have been possible to use Lemma 7.1 also in the proof of Theorem 1.3(a) in $\S 4$, since the maximal subgroups $N_{n}$ of $\Gamma^{+}=\Delta(p, q, r)$ constructed there are normalised by orientation-reversing elements of $\Gamma=\Delta[p, q, r]$. Instead of the natural representation of $\mathrm{PSL}_{2}(n)$, we could have used its representation on the cosets of a maximal subgroup $H \cong A_{5}$ for $n \equiv \pm 1 \bmod (5)$, or $H \cong S_{4}$ for $n \equiv \pm 1 \bmod (8)$ : in both of these cases there are two conjugacy classes of subgroups $H$, transposed by conjugation in $\mathrm{PGL}_{2}(n)$ (see [11, Ch. XII]) and corresponding to a chiral pair of hypermaps. However, in either case the point stabilisers $H$ have constant order as $n \rightarrow \infty$, whereas Proposition 3.2 requires $\left|N_{n}: K_{n}\right|=|H|$ to be unbounded, so we would need an alternative argument to show that $M$ is not normal in $\Gamma^{+}$, as in the proof of Proposition 3.3.

\section{Countable superabundance of some cocompact triangle groups}

Theorem 1.3(b) proves countable superabundance only for non-cocompact hyperbolic triangle groups $\Delta(p, q, r)$ and $\Delta[p, q, r]$. We would like to extend to this property to the cocompact case. The arguments we used to prove Theorem 1.3(b) depend on a standard generator of $\Delta(p, q, r)(Z$, without loss of generality) having infinite order, so that it can 
have a cycle $C$ of infinite length in some permutation representation, which is then proved to be primitive by identifying $C$ with $\mathbb{Z}$. Clearly this is impossible in the cocompact case, so a different approach is needed. The following is a first step in this direction.

Proposition 8.1. If one of $p, q$ and $r$ is even, another is divisible by 3, and the third is at least 7 , then the cocompact triangle groups $\Delta(p, q, r)$ and $\Delta[p, q, r]$ and their associated categories are countably superabundant.

Proof. By permuting periods and applying Lemma 3.1 we may assume that $p=3, q=2$ and $r \geq 7$. First suppose that $r=7$. We will construct an infinite transitive permutation representation of the group

$$
\Gamma=\Delta[3,2,7]=\left\langle X, Y, T \mid X^{3}=Y^{2}=T^{2}=(X Y)^{7}=(X T)^{2}=(Y T)^{2}=1\right\rangle
$$

(where $T=R_{2}$ ) in which the subgroup $\Gamma^{+}=\Delta(3,2,7)=\langle X, Y\rangle$ acts primitively, and we will then apply Proposition 3.3 to a point-stabiliser in $\Gamma^{+}$. This representation is constructed by adapting the Higman-Conder technique of 'sewing coset diagrams together', used in [7] to realise finite alternating and symmetric groups as quotients of $\Gamma^{+}$and $\Gamma$. We refer the reader to [7] for full technical details of this method. (Note that we have changed Conder's notation, which has $X^{2}=Y^{3}=1$, by transposing the symbols $X$ and $Y$; this has no significant effect on the following proof.)
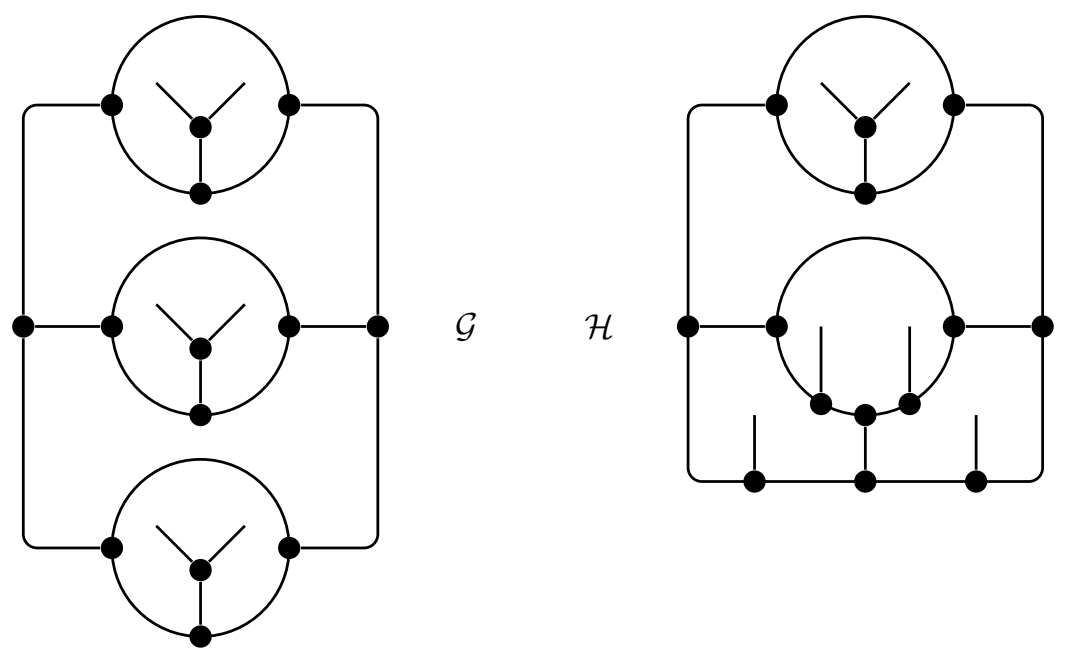

Figure 4: The maps $\mathcal{G}$ and $\mathcal{H}$.

Conder gives 14 coset diagrams $A, \ldots, N$ for subgroups of index $n=14, \ldots, 108$ in $\Gamma^{+}$, with respect to the generators $X$ and $Y$; these can be interpreted as describing transitive representations of $\Gamma^{+}$of degree $n$. Each diagram is bilaterally symmetric, so this action of $\Gamma^{+}$extends to a transitive representation of $\Gamma$ of degree $n$, with $T$ fixing vertices on the vertical axis of symmetry, and transposing pairs of vertices on opposite sides of it. Although Conder does not do this, in the spirit of the proof of Theorem 5.1 we can convert each of his diagrams into a planar map of type $\{7,3\}$ (equivalently a hypermap of type $(3,2,7)$ ) by contracting the small triangles representing 3 -cycles of $X$ to trivalent vertices, so that 
the cycles of $X, Y$ and $Z$ on directed edges correspond to its vertices, edges and faces. (Warning: although $\Gamma^{+}$acts as the monodromy group of this oriented map, permuting directed edges as described in Example 2 of $\S 2, \Gamma$ does not act as the monodromy group of the unoriented map, as in Example 1: the latter permutes flags, whereas $T$ uses the symmetry of the map to extend the action of $\Gamma^{+}$on directed edges to an action of $\Gamma$ on directed edges.)

We will construct an infinite coset diagram from Conder's diagrams $G$ and $H$ of degree $n=42$; the corresponding maps $\mathcal{G}$ and $\mathcal{H}$ are shown in Figure 4. Conder defines a (1)-handle in a diagram to be a pair $\alpha, \beta$ of fixed points of $Y$ with $\beta=\alpha X=\alpha T$, represented in the corresponding map by two free edges incident with the same vertex on the axis of symmetry. Thus $\mathcal{G}$ has three (1)-handles, while $\mathcal{H}$ has one. If diagrams $D_{i}(i=1,2)$ of degree $n_{i}$ have (1)-handles $\alpha_{i}, \beta_{i}$ then one can form a new diagram, called a (1)-join $D_{1}(1) D_{2}$, by replacing these four fixed points of $Y$ with transpositions $\left(\alpha_{1}, \alpha_{2}\right)$ and $\left(\beta_{1}, \beta_{2}\right)$, and leaving the permutations representing $X, Y$ and $T$ in $D_{1}$ and $D_{2}$ otherwise unchanged; the result is a new coset diagram giving a transitive representation of $\Gamma$ of degree $n_{1}+n_{2}$. In terms of the corresponding maps $\mathcal{D}_{i}$, this is a connected sum operation, in which the two surfaces are joined across cuts between the free ends of the free edges representing the fixed points $\alpha_{i}$ and $\beta_{i}$; in particular, if $\mathcal{D}_{i}$ has genus $g_{i}$ then $\mathcal{D}_{1}(1) \mathcal{D}_{2}$ has genus $g_{1}+g_{2}$. This is illustrated in Figure 5, where the (1)-handle at the bottom of $\mathcal{G}$ is joined to that at the top of $\mathcal{H}$ by two dashed edges to form $\mathcal{G}(1) \mathcal{H}$; these edges can be carried by a tube connecting the two surfaces, showing the additivity of the genera (both equal to 0 here). (Further details about this and more general joining operations on dessins can be found in [27].)

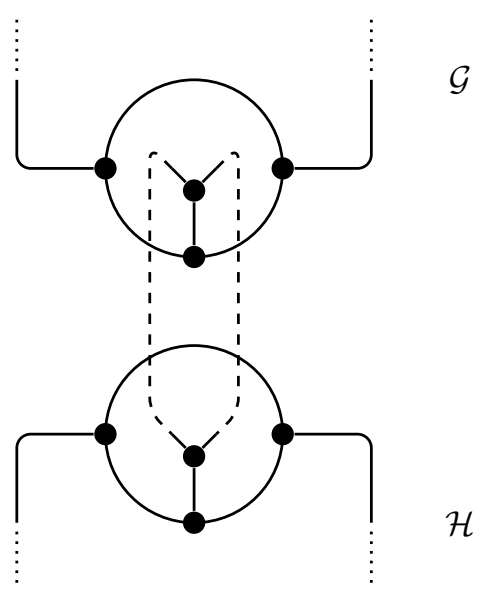

Figure 5: Joining $\mathcal{G}$ and $\mathcal{H}$ to form $\mathcal{G}(1) \mathcal{H}$.

Using (1)-handles in $G$ and $H$, we first form an infinite diagram

$$
H(1) G(1) G(1) G(1) G \cdots
$$

corresponding to an infinite planar map $\mathcal{H}(1) \mathcal{G}(1) \mathcal{G}(1) \mathcal{G}(1) \mathcal{G} \cdots$ of type $\{7,3\}$ : the $(1)$ handle at the top of each map $\mathcal{H}$ or $\mathcal{G}$ is joined, as in Figure 5, to that at the bottom of the next map $\mathcal{G}$. In this chain, each copy of $\mathcal{G}$ has an unused (1)-handle; we join these 
arbitrarily in pairs, using (1)-compositions. Each such join adds a bridge to the underlying surface, increasing the genus by 1 , so the result is an oriented trivalent map $\mathcal{N}$ of type $\{7,3\}$ and of infinite genus. This gives an infinite transitive permutation representation $X \mapsto x$, $Y \mapsto y, T \mapsto t$ of $\Gamma$ on the directed edges of $\mathcal{N}$, with $\Gamma^{+}$again acting as its monodromy group, and $T$ acting as a reflection.

We need to prove that $\Gamma$ and $\Gamma^{+}$act primitively. As shown by Conder [7] the permutation $w=y x t$ (=xyt in his notation) induced by $Y X T$ has cycle structures $1^{3} 13^{3}$ and $1^{1} 3^{1} 10^{1} 11^{1} 17^{1}$ in $G$ and $H$. In each of the (1)-compositions we have used, two fixed points of $w$ are paired to form a cycle of length 2 of $w$, and a cycle of $w$ of length 13 in $G$ is merged with one of length 13 or 10 in $G$ or $H$ to form a cycle of length 26 or 23 . All other cycles of $w$ are unchanged, so in particular its cycle $C$ of length 17 in $H$ remains a cycle in the final diagram. Since all other cycles of $w$ have finite length coprime to 17, some power of $w$ acts on $C$ as $w$ and fixes all other points. Since 17 is prime, it follows that if $\Gamma^{+}$acts imprimitively, then all points in $C$ must lie in the same equivalence class $E$. Now $C$ is what Conder calls a 'useful cycle', since it contains a fixed point of $y$ not in a (1)-handle (the right-hand free edge $\beta$ in the central circle in $\mathcal{H}$ in Figure 4 ) and a pair of points from a 3-cycle of $x$ (namely $\beta$ and $\beta x=\beta w^{8}$ ). It follows that $X$ and $Y$ leave $E$ invariant, which is impossible since they generate the transitive group $\Gamma^{+}$. Thus $\Gamma^{+}$acts primitively (as therefore does $\Gamma$ ), so the point-stabilisers $N=\Gamma_{\alpha}$ and $N^{+}=\Gamma_{\alpha}^{+}$of a directed edge $\alpha$ are maximal subgroups of $\Gamma$ and $\Gamma^{+}$. By the Reidemeister-Schreier algorithm, $N^{+}$is a free product of four cyclic groups of order 2 (arising from fixed points of $y$ in $H$ not in the (1)-handle), and infinitely many of infinite order (two arising from each bridge between a pair of copies of $G$ ). Thus $N^{+}$admits an epimorphism onto $F_{\infty}$, so Proposition 3.3 shows that $\Gamma^{+}$is countably abundant.

We can choose $\alpha$ to be fixed by $t$, so that $T \in N$, and hence $N$ is a semidirect product of $N^{+}$by $\langle T\rangle$. The action of $t$ is to reflect $H$ and all the copies of $G$ in the diagram, together with the bridges added between pairs of them. Acting by conjugation on $\mathrm{N}^{+}$, $T$ therefore induces two transpositions on the elliptic generators of order 2. Each bridge contributes a pair of free generators to $\mathrm{N}^{+}$, one of them (represented by a loop crossing the bridge and returning 'at ground level'), centralised by $T$, the other (represented by a loop transverse to the first, following a cross-section of the bridge) inverted by $T$; by sending $T$, together with the inverted generators and the four elliptic generators of $N^{+}$, to the identity, we can map $N$ onto the free group of countably infinite rank generated by the centralised generators, so Proposition 3.3 shows that $\Gamma$ is countably abundant. In fact, there are $2^{\aleph_{0}}$ ways of pairing the copies of $G$ to produce bridges, giving mutually inequivalent permutation representations and hence mutually non-conjugate subgroups $N$ and $N^{+}$of $\Gamma$ and $\Gamma^{+}$, so this argument establishes countable superabundance.

The extension to the case $r \geq 7$ is essentially the same, but based on the coset diagrams in Conder's later paper [8] on alternating and symmetric quotients of $\Delta(3,2, r)$ and $\Delta[3,2, r]$. In this case his coset diagrams $S(h, d)$ and $U(h, d)$ play the roles of $G$ and $H$, where $r=h+6 d$ with $d \in \mathbb{N}$ and $h=7, \ldots, 12$.

Proposition 8.1 accounts for a proportion $121 / 216$ of all hyperbolic triples. It seems plausible that coset diagrams of Everitt [13] and others, constructed to extend Conder's results on alternating group quotients to all finitely generated non-elementary Fuchsian groups, could be used to prove that all cocompact hyperbolic triangle groups $\Delta(p, q, r)$ and $\Delta[p, q, r]$, together with their associated categories, are countably superabundant, thus proving Conjecture 1.4. 


\section{Realisation of other groups}

Finally, although this paper is mainly about triangle groups and their associated categories of maps and hypermaps, we can deduce realisation properties for many other groups and categories.

Theorem 9.1. If a group $\Gamma$ has an epimorphism onto a non-abelian free group then it is finitely and countably superabundant.

Proof. By Theorem 1.3 the free group $F_{2}=\Delta(\infty, \infty, \infty)$ has these properties. Since $F_{2}$ is an epimorphic image of every other non-abelian free group, the result follows from Lemma 3.1.

For example, Theorem 9.1 applies to the fundamental groups $\Gamma$ of many topological spaces, so their categories of coverings are finitely and countably superabundant.. Examples include compact orientable surfaces of genus $g$ with $k$ punctures, where $2 g+k \geq 3$. Taking $g=0, k=3$ shows that the category $\mathfrak{D}$ of all dessins is finitely superabundant (see Theorem 1.3(a) for a more specific result); in fact, Cori and Machì [9] proved that every finite group is the automorphism group of a finite oriented hypermap, two years before Grothendieck introduced dessins in [20].

Hidalgo [21] has proved the stronger result that every action of a finite group $A$ by orientation-preserving self-homeomorphisms of a compact oriented surface $S$ is topologically equivalent to the automorphism group of a dessin. One way to see this is to triangulate $S / A$, with all critical values of the projection $\pi: S \rightarrow S / A$ among the vertices, none of which has valency 1 , and then to add an edge to an additional 1-valent vertex $v$ in the interior of a face. This gives a map (that is, a dessin with $q=2$ ) on $S / A$ which lifts via $\pi$ to a dessin $\mathcal{D}$ on $S$ with $A \leq \operatorname{Aut}(\mathcal{D})$. The only 1 -valent vertices in $\mathcal{D}$ are the $|A|$ vertices in $\pi^{-1}(v)$. These are permuted by $\operatorname{Aut}(\mathcal{D})$, with $A$ and hence $\operatorname{Aut}(\mathcal{D})$ acting transitively; however, the stabiliser of a 1-valent vertex (in any dessin) must be the identity, so $\operatorname{Aut}(\mathcal{D})=A$, as required. By starting with inequivalent triangulations of $S / A$ one can obtain infinitely many non-isomorphic dessins realising this action of $A$.

\section{ORCID iD}

Gareth A. Jones (iD https://orcid.org/0000-0002-7082-7025

\section{References}

[1] L. Babai, On the abstract group of automorphisms, in: H. N. V. Temperley (ed.), Combinatorics, Cambridge University Press, Cambridge-New York, volume 52 of London Mathematical Society Lecture Note Series, pp. 1-40, 1981, doi:10.1017/cbo9780511662157.003, proceedings of the Eighth British Combinatorial Conference held at University College, Swansea, July 20 24, 1981.

[2] L. Babai, Automorphism groups, isomorphism, reconstruction, in: R. L. Graham, M. Grötschel and L. Lovász (eds.), Handbook of Combinatorics, Volume 2, Elsevier, Amsterdam, pp. 14471540, 1995, doi:10.5555/233228.233236.

[3] M. Belolipetsky and A. Lubotzky, Finite groups and hyperbolic manifolds, Invent. Math. 162 (2005), 459-472, doi:10.1007/s00222-005-0446-z.

[4] G. Birkhoff, Sobre los grupos de automorfismos, Rev. Un. Mat. Argentina 11 (1946), 155-157, https://inmabb.criba.edu .ar/revuma/revuma.php?p=toc/vol11. 
[5] R. Bottinelli, L. Grave de Peralta and A. Kolpakov, Telescopic groups and symmetries of combinatorial maps, Algebr. Comb. 3 (2020), 483-508, doi:10.5802/alco.101.

[6] J. L. Brenner and R. C. Lyndon, Maximal nonparabolic subgroups of the modular group, Math. Ann. 263 (1983), 1-11, doi:10.1007/bf01457079.

[7] M. D. E. Conder, Generators for alternating and symmetric groups, J. London Math. Soc. 22 (1980), 75-86, doi:10.1112/jlms/s2-22.1.75.

[8] M. D. E. Conder, More on generators for alternating and symmetric groups, Quart. J. Math. Oxford Ser. 32 (1981), 137-163, doi:10.1093/qmath/32.2.137.

[9] R. Cori and A. Machì, Construction of maps with prescribed automorphism group, Theoret. Comput. Sci. 21 (1982), 91-98, doi:10.1016/0304-3975(82)90090-1.

[10] H. S. M. Coxeter and W. O. J. Moser, Generators and Relations for Discrete Groups, volume 14 of Ergebnisse der Mathematik und ihrer Grenzgebiete, Springer-Verlag, New York-Heidelberg, 3rd edition, 1972, doi:10.1007/978-3-662-21946-1.

[11] L. E. Dickson, Linear Groups: With an Exposition of the Galois Field Theory, Dover Publications, New York, 1958.

[12] J. P. Doignon, A convex polytope and an antimatroid for any given, finite group, Electron. Notes Discrete Math. 54 (2016), 21-25, doi:10.1016/j.endm.2016.09.005.

[13] B. Everitt, Alternating quotients of Fuchsian groups, J. Algebra 223 (2000), 457-476, doi: 10.1006/jabr.1999.8014.

[14] E. Fried and J. Kollár, Automorphism groups of algebraic number fields, Math. Z. 163 (1978), 121-123, doi:10.1007/bf01214058.

[15] R. Frucht, Herstellung von Graphen mit vorgegebener abstrakter Gruppe, Compositio Math 6 (1939), 239-250, http: / /www.numdam.org/item?id=CM_1939_6_239_o.

[16] S. Garion, On Beauville structures for $\mathrm{PSL}_{2}(q)$, J. Group Theory 18 (2015), 981-1019, doi: 10.1515/jgth-2015-0020.

[17] E. Girondo and G. González-Diez, Introduction to Compact Riemann Surfaces and Dessins d'Enfants, volume 79 of London Mathematical Society Student Texts, Cambridge University Press, Cambridge, 2012, doi:10.1017/cbo9781139048910.

[18] L. Greenberg, Conformal transformations of Riemann surfaces, Amer. J. Math. 82 (1960), 749760, doi:10.2307/2372937.

[19] L. Greenberg, Maximal groups and signatures, in: L. Greenberg (ed.), Discontinuous Groups and Riemann Surfaces, volume 79 of Annals of Mathematics Studies, 1974 pp. 207-226, doi:10. 1515/9781400881642-017, proceedings of the 1973 Conference at the University of Maryland, College Park, Maryland, May 21 - 25, 1973.

[20] A. Grothendieck, Esquisse d'un programme, in: Geometric Galois Actions, Volume 1, Cambridge University Press, Cambridge, volume 242 of London Mathematical Society Lecture Note Series, pp. 5-48, 1997, doi:10.1017/cbo9780511758874.003.

[21] R. A. Hidalgo, Automorphism groups of dessins d'enfants, Arch. Math. (Basel) 112 (2019), 13-18, doi:10.1007/s00013-018-1222-9.

[22] A. H. M. Hoare, Subgroups of N.E.C. groups and finite permutation groups, Quart. J. Math. Oxford Ser. 41 (1990), 45-59, doi:10.1093/qmath/41.1.45.

[23] G. A. Jones, Exotic behaviour of infinite hypermaps, Ars Math. Contemp. 1 (2008), 51-65, doi:10.26493/1855-3974.24.035.

[24] G. A. Jones, Combinatorial categories and permutation groups, Ars Math. Contemp. 10 (2016), 237-254, doi:10.26493/1855-3974.545.fd5. 
[25] G. A. Jones, Maximal subgroups of the modular and other groups, J. Group Theory 22 (2019), 277-296, doi:10.1515/jgth-2018-0144.

[26] G. A. Jones, Automorphism groups of maps, hypermaps and dessins, Art Discrete Appl. Math. 3 (2020), \#P1.06, doi:10.26493/2590-9770.1275.e77.

[27] G. A. Jones, Joining Dessins together, in: Galois Covers, Grothendieck-Teichmüller Theory and Dessins d'Enfants, Springer, Cham, volume 330 of Springer Proceedings in Mathematics \& Statistics, pp. 121-154, 2020, doi:10.1007/978-3-030-51795-3_7.

[28] G. A. Jones and D. Singerman, Theory of maps on orientable surfaces, Proc. London Math. Soc. 37 (1978), 273-307, doi:10.1112/plms/s3-37.2.273.

[29] G. A. Jones and J. Wolfart, Dessins d'Enfants on Riemann Surfaces, Springer Monographs in Mathematics, Springer, Cham, 2016, doi:10.1007/978-3-319-24711-3.

[30] R. S. Kulkarni, Geometry of Neumann subgroups, J. Austral. Math. Soc. Ser. A 47 (1989), 350-367, doi:10.1017/s1446788700033085.

[31] S. K. Lando and A. K. Zvonkin, Graphs on Surfaces and Their Applications, volume 141 of Encyclopaedia of Mathematical Sciences, Springer-Verlag, Berlin, 2004, doi:10.1007/ 978-3-540-38361-1.

[32] R. C. Lyndon and P. E. Schupp, Combinatorial Group Theory, Classics in Mathematics, Springer-Verlag, Berlin, 2001, doi:10.1007/978-3-642-61896-3.

[33] W. Magnus, Rational representations of Fuchsian groups and non-parabolic subgroups of the modular group, Nachr. Akad. Wiss. Göttingen Math.-Phys. Kl. II (1973), 179-189.

[34] W. Magnus, Noneuclidean Tesselations and Their Groups, volume 9 of Pure and Applied Mathematics, Academic Press, New York-London, 1974.

[35] W. S. Massey, Algebraic Topology: An Introduction, Graduate Texts in Mathematics, Harcourt, Brace \& World, New York, 1967.

[36] P. McMullen and E. Schulte, Abstract Regular Polytopes, volume 92 of Encyclopedia of Mathematics and its Applications, Cambridge University Press, Cambridge, 2002, doi:10.1017/ cbo9780511546686.

[37] E. Mendelsohn, Every group is the collineation group of some projective plane, J. Geom. 2 (1972), 97-106, doi:10.1007/bf01918417.

[38] E. Mendelsohn, On the groups of automorphisms of Steiner triple and quadruple systems, $J$. Comb. Theory Ser. A 25 (1978), 97-104, doi:10.1016/0097-3165(78)90072-9.

[39] J. R. Munkres, Topology, Prentice Hall, Upper Saddle River, NJ, 2nd edition, 2000.

[40] B. H. Neumann, Ueber ein gruppentheoretisch-arithmetisches Problem, S.B. Preuss. Akad. Wiss. Phys.-Math. Kl. (1933), 18.

[41] P. M. Neumann, The $S Q$-universality of some finitely presented groups, J. Austral. Math. Soc. 16 (1973), 1-6, doi:10.1017/s1446788700013859.

[42] A. J. Ol'šanskiı̌, An infinite group with subgroups of prime orders, Izv. Akad. Nauk SSSR Ser. Mat. 44 (1980), 309-321, doi:10.1070/im1981v016n02abeh001307.

[43] A. Y. Ol'shanskiı̆, On the Bass-Lubotzky question about quotients of hyperbolic groups, $J$. Algebra 226 (2000), 807-817, doi:10.1006/jabr.1999.8170.

[44] C. E. Praeger and C. Schneider, Permutation Groups and Cartesian Decompositions, volume 449 of London Mathematical Society Lecture Note Series, Cambridge University Press, Cambridge, 2018, doi:10.1017/9781139194006.

[45] G. Sabidussi, Graphs with given group and given graph-theoretical properties, Canadian J. Math. 9 (1957), 515-525, doi:10.4153/cjm-1957-060-7. 
[46] G. Sabidussi, Graphs with given infinite group, Monatsh. Math. 64 (1960), 64-67, doi:10.1007/ bf01319053.

[47] E. Schulte and G. I. Williams, Polytopes with preassigned automorphism groups, Discrete Comput. Geom. 54 (2015), 444-458, doi:10.1007/s00454-015-9710-1.

[48] D. Singerman, Subgroups of Fuschian groups and finite permutation groups, Bull. London Math. Soc. 2 (1970), 319-323, doi:10.1112/blms/2.3.319.

[49] D. Singerman, Finitely maximal Fuchsian groups, J. London Math. Soc. 6 (1972), 29-38, doi: 10.1112/jlms/s2-6.1.29.

[50] D. Singerman, Symmetries of Riemann surfaces with large automorphism group, Math. Ann. 210 (1974), 17-32, doi:10.1007/bf01344543.

[51] D. Singerman and R. I. Syddall, The Riemann surface of a uniform dessin, Beiträge Algebra Geom. 44 (2003), 413-430, https://www.emis.de/journals/BAG/vol.44/no. 2/9.html.

[52] K. Takeuchi, Arithmetic triangle groups, J. Math. Soc. Japan 29 (1977), 91-106, doi:10.2969/ jmsj/02910091.

[53] C. Tretkoff, Nonparabolic subgroups of the modular group, Glasgow Math. J. 16 (1975), 91102, doi:10.1017/s0017089500002573.

[54] T. R. S. Walsh, Hypermaps versus bipartite maps, J. Comb. Theory Ser. B 18 (1975), 155-163, doi:10.1016/0095-8956(75)90042-8. 